\title{
Atom lithography without laser cooling
}

\author{
B. Smeets • P. van der Straten • T. Meijer • \\ C.G.C.H.M. Fabrie • K.A.H. van Leeuwen
}

Received: 24 August 2009 / Revised version: 2 November 2009 / Published online: 19 December 2009

(c) The Author(s) 2009. This article is published with open access at Springerlink.com

\begin{abstract}
Using direct-write atom lithography, Fe nanolines are deposited with a pitch of $186 \mathrm{~nm}$, a full width at half maximum (FWHM) of $50 \mathrm{~nm}$, and a height of up to $6 \mathrm{~nm}$. These values are achieved by relying on geometrical collimation of the atomic beam, thus without using laser collimation techniques. This opens the way for applying directwrite atom lithography to a wide variety of elements.
\end{abstract}

\section{Introduction}

In direct-write atom lithography, also known as laserfocused deposition, atoms are focused into a periodic pattern by a standing light wave. This technique has been applied to $\mathrm{Na}$ [1], $\mathrm{Cr}$ [2,3], $\mathrm{Al}$ [4], $\mathrm{Yb}$ [5], and $\mathrm{Fe}[6,7]$. Just as for the case of focusing light, a well collimated atomic beam is crucial for atom lithography. An atomic beam arriving at the lens with a local angular spread $\alpha$ (see Fig. 1) will result in broadening of the deposited features. To obtain a well collimated atomic beam, laser cooling techniques are generally used. However, effective laser cooling requires a closed optical transition. For numerous elements a closed transition

\footnotetext{
B. Smeets · T. Meijer · C.G.C.H.M. Fabrie ·

K.A.H. van Leeuwen ( $₫)$

Department of Applied Physics, Eindhoven University

of Technology, P.O. Box 513, 5600 MB Eindhoven,

The Netherlands

e-mail: k.a.h.v.leeuwen@tue.nl

P. van der Straten

Atom Optics and Ultrafast Dynamics, Utrecht University,

P.O. Box 80000, 3508 TA Utrecht, The Netherlands

Present address:

B. Smeets

ASML, P.O. Box 324, 5500 AH Veldhoven, The Netherlands
}

is not accessible with present lasers, or hyperfine splitting is present, and the use of several repumping lasers becomes a necessity. For Fe, for instance, an accessible closed transition from the ground state does not exist, resulting in the loss of focusable atoms when applying laser cooling [8].

It is because of these drawbacks that we decided to laser focus Fe without the use of any laser collimation techniques, simply relying for this part on geometrical collimation. We will show that in this way periodic grids of Fe nanolines with a FWHM of $50 \mathrm{~nm}$, a height of up to $6 \mathrm{~nm}$, and a period of $186 \mathrm{~nm}$ can still be deposited. Thus, we prove that atoms can be focused without using laser collimation. This makes the technique suitable for direct-write atom lithography of any species with transitions of sufficient strength that are not necessarily closed. Especially for atom lithography of technologically relevant elements such as Ga [9], In [10, 11], and Si [12], for which laser collimation imposes large experimental difficulties, this is a step forward. Also, laser focusing of molecules may be feasible this way.

For earlier reviews of both direct-write and resist-based atom lithography, see [13, 14].

As the experiments are time-consuming, it is important to choose the experimental parameters carefully a priori. This is facilitated by the availability of an efficient and realistic simulation procedure for the atomic trajectories. Also, a comparison with simulations is essential for the careful analysis of the experimental results. We use a fast semiclassical Monte-Carlo procedure based on the dressed-state model [15] that includes the effects of diffusion induced by spontaneous emission as well as of non-adiabatic transitions between dressed states.

In Sect. 2 our procedure for simulating standing-wave laser focusing is described in more detail. In Sect. 3 the experimental setup is described. The experimental results of atom lithography without laser collimation are compared 


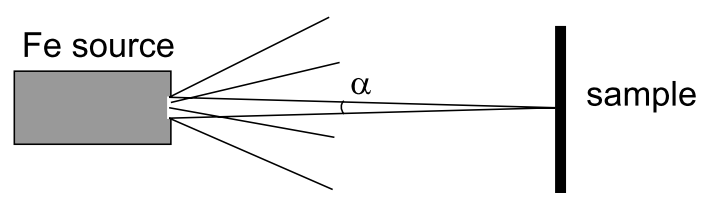

Fig. 1 Geometrical collimation. At each spot on the substrate, atoms arrive with an angular spread $\alpha$, defined by the nozzle size and the distance between source and sample

with the outcome of the simulations in Sect. 4, followed by the conclusions in Sect. 5.

An analysis of the magnetic properties of the deposited nanolines will be published elsewhere [16].

\section{Simulations}

Our simulation is based on the dressed-state model, introduced by Dalibard and Cohen-Tannoudji [15]. In this model one considers the eigenstates of the complete atom plus laser light field system. The energies of these states are given by

$$
\begin{aligned}
& E_{n,+}=(n+1) \hbar \omega_{L}-\frac{\hbar \Delta}{2}+\frac{\hbar \Omega}{2}, \\
& E_{n,-}=(n+1) \hbar \omega_{L}-\frac{\hbar \Delta}{2}-\frac{\hbar \Omega}{2},
\end{aligned}
$$

with $n$ the number of photons in the light field, $\omega_{L}$ the frequency of the light field, $\Delta=\omega_{L}-\omega_{0}$ the detuning of the light field from the atomic resonance $\omega_{0}$. The frequency separation between the dressed states $\Omega$ is given by

$\Omega=\sqrt{\omega_{R}^{2}+\Delta^{2}}$,

with $\omega_{R}=\Gamma \sqrt{I / 2 I_{S}}$ the Rabi frequency, where $\Gamma$ represents the natural linewidth, $I$ the light intensity, and $I_{S}$ the saturation intensity. The dressed states are a linear superposition of the ground and excited states:

$$
\begin{aligned}
& |n,+\rangle=\cos (\theta)|e, n\rangle+\sin (\theta)|g, n+1\rangle, \\
& |n,-\rangle=-\sin (\theta)|e, n\rangle+\cos (\theta)|g, n+1\rangle .
\end{aligned}
$$

Here $\theta$ is defined by

$\cos (2 \theta)=-\Delta / \Omega$.

In our simulation, atoms move as classical point particles in a potential field given by the dressed-state energy shift $\pm \frac{\hbar \Omega(\mathbf{r})}{2}$, where the sign indicates the dressed state the system is currently in. The light mask is described as a onedimensional Gaussian standing wave along the $x$-axis with a waist $w$ and maximum on-axis intensity $I_{0}$ :

$I(\mathbf{r})=I_{0} \sin ^{2}(k x) \exp \left(-2 \frac{z^{2}}{w^{2}}\right)$.

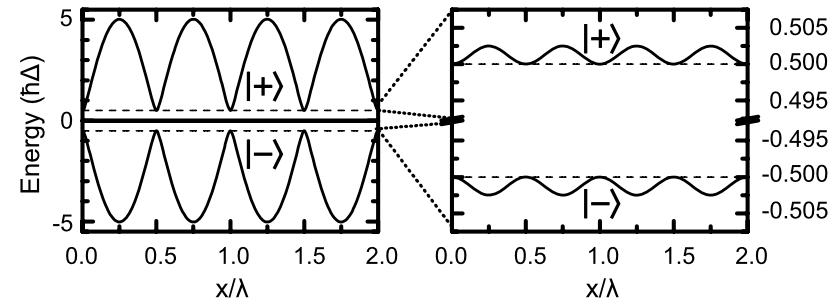

Fig. 2 Left: dressed-state potentials for $\omega_{R} /|\Delta|=10$. Dashed lines indicate zero-intensity levels. Note the difference in shape between the potential minima for $|+\rangle$ and $|-\rangle$ states. Right: same, for $\omega_{R} /|\Delta|=0.1$. In this case both potentials are equivalent

Here $k=2 \pi / \lambda$ is the wavenumber of the light and the propagation direction of the atoms is assumed to be along the $z$-axis. As the atoms' kinetic energy is much larger than the potential height of the light mask we can neglect its effect on the longitudinal motion, so that the atom's motion is described by a one-dimensional Newtonian equation of motion in the $x$-direction, which is solved numerically.

Outside the standing wave the intensity $I$ of the light is zero and the ground state is $|+\rangle$ for $\Delta>0$ and $|-\rangle$ for $\Delta<0$. Unless the atoms undergo a spontaneous or nonadiabatic transition, they will stay in their initial state.

The effects of spontaneous emission, and hence diffusion in the atom's motion, are included in the model by evaluating the probability that an atom has undergone spontaneous emission at regular time intervals. By far the largest contribution to the diffusion is caused by emissions that change the sign of the dressed state, causing an instant reversal of the force on the atom. The relevant transition rates are given by

$\Gamma_{+-}=\Gamma \cos ^{4} \theta$

$\Gamma_{-+}=\Gamma \sin ^{4} \theta$,

with $\theta$ defined in (4). The probability of a dressed-state transition due to spontaneous emission is given by $\tau \Gamma_{+-}$or $\tau \Gamma_{-+}$, depending on the initial dressed state, with $\tau$ the time interval.

Non-adiabatic transitions can occur when the atom moves too fast, or in other words, the eigenstates (dressed states) change too fast for the internal state of the atom to follow. The atom is then transferred from one dressed state to the other. This is most likely to occur when atoms cross a node of the standing wave. Non-adiabatic transfer is incorporated in the simulation procedure, but does not contribute significantly for present experimental conditions.

Before we show the results of these semiclassical simulations, we discuss the potential that is used. The left part of Fig. 2 displays the potential for the case of a strong light field, where $\omega_{R} /|\Delta|=10$. The case of a weak light field with $\omega_{R} /|\Delta|=0.1$ is displayed to the right. Both potentials are very different in shape. 
In the strong field limit, the potential minima of the $|+\rangle$ state differ radically in shape from those of the $|-\rangle$ state. The minima of the $|-\rangle$ state are smooth, broad and parabolic over a large range around the minima. Using such a potential is a way to avoid non-parabolic aberrations in the atom focusing process. On the other hand, the minima of the $|+\rangle$ state look like a V-shaped potential. The oscillation period of the atoms in the potential then depends on the starting point of the oscillation, leading to strong aberration in the focussing. The optical equivalent of such a lens is a conical lens or axicon. The aberration leads to a less sharply defined focus with a long focal depth, or a focal line.

For positive detuning, the atoms start out in the $|+\rangle$ state and are subject to the V-shaped potential. Provided that they do not switch between dressed states due to spontaneous emission, this then creates focal lines-aligned with the nodes - rather than focal points. Hence, the formation of structures should be fairly insensitive to the experimental parameters. For negative detuning, the situation is reversed and the atoms are focussed by the smooth potential, in principle creating sharper focal points-aligned with the antinodesbecause of the reduced non-parabolic aberration. However, these focal points are subject to severe aberration caused by the spread in longitudinal velocity, the atom-optical equivalent of chromatic aberration. This negates the advantage of the reduced non-parabolic aberration.

The potential for a weak standing wave is sine-like. The difference in shape between the $|+\rangle$ and $|-\rangle$ state minima has vanished, and focusing is expected to proceed in a similar fashion for both states.

The experiments and simulations described here have all been performed in the strong field case, with typical maximum values of $\omega_{R} /|\Delta| \approx 5$, with both positive and negative detuning.

For the experiment, the ${ }^{5} \mathrm{D}_{4} \rightarrow{ }^{5} \mathrm{~F}_{5}$ atomic transition of iron is used. The wavelength is $372 \mathrm{~nm}$. The isotope ${ }^{56} \mathrm{Fe}$ has a natural abundance of $91.8 \%$, of which at a typical evaporation temperature of $2000 \mathrm{~K} \approx 50 \%$ is still in the ${ }^{5} \mathrm{D}_{4}$ ground state. Hyperfine structure is absent in ${ }^{56} \mathrm{Fe}$. For this transition, $\Gamma=1.62 \times 10^{7} \mathrm{~s}^{-1}$ and the saturation intensity for the $M_{g}=4 \rightarrow M_{e}=5$ magnetic subtransition is $I_{s}=65 \mathrm{~W} / \mathrm{m}^{2}$. The light mask is a one-dimensional standing light wave produced by an elliptical Gaussian beam with waist radius $w_{x}$ along the atomic beam axis and $w_{y}$ perpendicular to it.

For the calculation, we start with 10,000 atoms. Each atom is initially assumed to be in a random magnetic substate. The saturation intensity is then calculated using the appropriate Clebsch-Gordan coefficient for the substate and laser polarization. Spatially, the atoms are homogeneously distributed over a single wavelength in the $x$-direction.

The longitudinal velocity distribution of the atomic beam is that of an effusive beam, i.e., a Maxwell-Boltzmann dis- tribution with average velocity $\langle v\rangle=\sqrt{\frac{8 k_{B} T}{\pi m}}$. The transverse velocity distribution of the atoms is determined by the longitudinal velocity and the geometrical collimation. The beam divergence, emerging from a round nozzle with diameter $D$ at a distance $L$ from the standing wave, is characterized in good approximation by a Gaussian angular distribution with a root-mean-square (RMS) width of $\frac{D}{4 L}$. This leads to a Gaussian transverse velocity distribution with an RMS spread of $\frac{D}{4 L}\langle v\rangle$.

The equation of motion of every atom is integrated over a set time interval, before we check for spontaneous and non-adiabatic transitions. The effect of both transitions is to change from one dressed state to the other. The possibility that atoms, which undergo a spontaneous emission, decay to a different magnetic substate, is neglected.

The integration starts when the atom's longitudinal position $(z)$ is three times the waist radius of the Gaussian laser beam before the center of the laser beam, and ends at the same distance after the center. We make a histogram of the atomic flux distribution at set longitudinal positions.

To demonstrate the difference in focusing between the negative and positive detuning, we first simulate focusing of a perfectly collimated atom beam, setting the RMS spread in transverse velocity to zero. A round laser beam with $w_{y}=$ $w_{z}=50 \mu \mathrm{m}$, a power of $50 \mu \mathrm{mW}$ and linear polarization is taken. The detuning of the light with respect to the atomic transition is set at $\Delta= \pm 150(2 \pi) \mathrm{MHz}( \pm 58 \Gamma)$. These parameters lead to a maximum value of $\omega_{R} /|\Delta| \approx 5.9$. The results are shown in Fig. 3. Interaction with a red-detuned standing wave generates focal points, as shown on the left. The blue-detuned standing wave gives rise to focal lines, as shown on the right. For blue detuning, the atoms experience on average 0.45 dressed-state-changing spontaneous emissions occur. For red detuning, the average number of emissions is 0.7 , reflecting the fact that atoms are attracted to intensity maxima in this case, where the absorption rate is higher.

The strength of the lenses varies per atom. This is due primarily to the distribution over the magnetic substates of the Fe atoms; secondarily, the longitudinal velocity spread of the Fe atoms also contributes. The relatively long $z$-range over which atom focusing occurs for the blue-detuned case stands out.

\section{Experimental setup}

An Fe atomic beam is produced using an $\mathrm{Al}_{2} \mathrm{O}_{3}$ crucible with a nozzle diameter of $1 \mathrm{~mm}$ heated to a temperature of around $2,000 \mathrm{~K}$ by a carbon spiral heater [17], resulting in a typical Fe density in the source of $n_{\mathrm{Fe}}=4 \times 10^{20} \mathrm{~m}^{-3}$. At this temperature the Fe-beam intensity is $I_{\mathrm{Fe}}=2.5 \times$ 


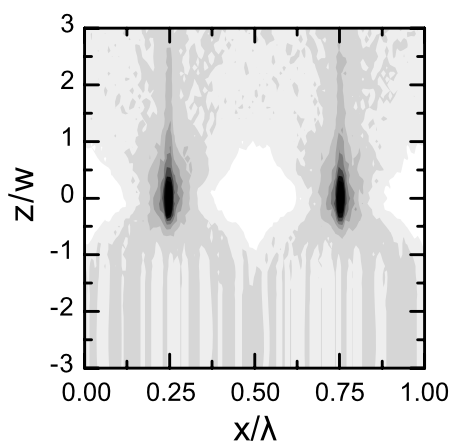

Fig. 3 Flux distribution of an atomic beam passing from below through a strong light mask $\omega_{R} /|\Delta|=10$. Left: $\Delta<0$ generates focal points, i.e., the size of the focus is approximately one beam waist in the

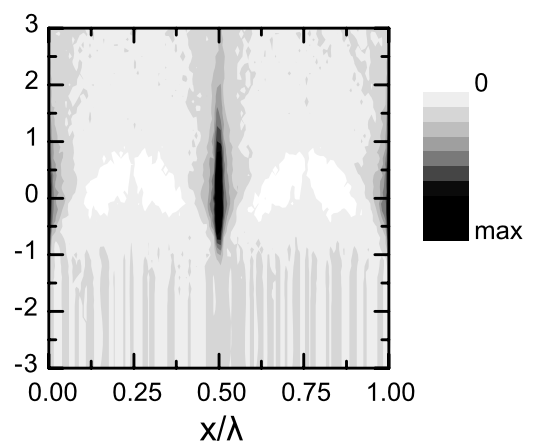

$z$-direction. Right: $\Delta>0$ results in focal lines, i.e., the focus is spread over more than two beam waists. This difference in flux distribution is due to the difference in potential as shown on the left side of Fig. 2
Fig. 4 Left: sample holder. Right: cross section of sample holder. The Si sample is clamped to a ceramic isolator that is screwed to the steel frame. The mirror is pressed to the frame by a spring

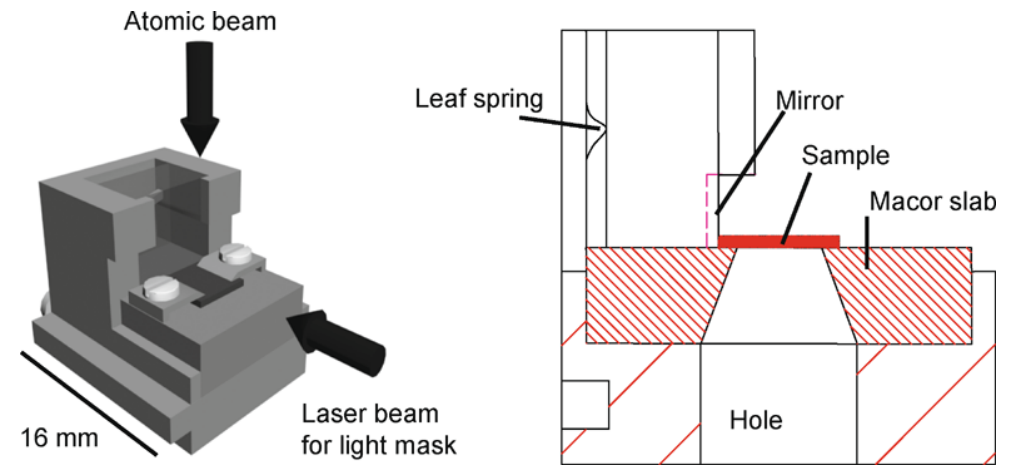

(Fig. 4), on which also an $8 \times 8 \times 3 \mathrm{~mm}$ mirror is mounted. These sample holders can easily be removed from the deposition chamber and stored in a load lock by a magnetic translator so that the alignment of sample and mirror, which have to be perpendicular to each other within $1 \mathrm{mrad}$, can be done ex vacuo. The pressure in the deposition chamber is typically $1 \times 10^{-8}$ mbar.

To create the standing light wave, the laser beam is focused to a waist of $50 \mu \mathrm{m}$ parallel to the atomic beam and $80 \mu \mathrm{m}$ perpendicular to the atomic beam, located at the surface of the sample holder mirror. The light is linearly polarized.

The substrate is positioned close to the center of the laser beam. Experiments have been performed with the substrate at the center of the waist, thus cutting the laser beam in half, as well as with the substrate positioned at the rear end of the laser beam waist, such that just $10 \%$ of the power in the laser beam is cut off by the substrate. Both cases are schematically drawn in Fig. 5. According to the simulations, the 50\% cut-off configuration should lead to somewhat narrower focussed lines and results that are less sensitive to variations in laser power. However, diffraction effects, which are difficult to take into account in the calculations, are more serious than in the $10 \%$ cut-off case.

To prevent Fe from oxidizing, a Ag capping layer of approximately $5 \mathrm{~nm}$ is deposited using an effusive source op- 
erated at a typical temperature of $1,140 \mathrm{~K}$, resulting in a deposition rate of $0.15 \mathrm{~nm} / \mathrm{min}$.

Although the height of the deposited structures can be easily measured by an AFM microscope, in order to determine the average thickness of the deposited Fe layer as well (and hence the thickness of the background layer) we need to calibrate the overall $\mathrm{Fe}$ deposition rate. To achieve this, we first produce structures by depositing Fe through a mechanical mask. The mask is made by e-beam lithography of a SiN membrane. Lines have been etched in the membrane with a width of about $150 \mathrm{~nm}$ and a period of $744 \mathrm{~nm}$ over an area of $250 \times 250 \mu \mathrm{m}$. The mask is pressed onto the substrate with a metal foil of $100 \mu \mathrm{m}$ as spacer between the bottom of the mask and the front of the substrate. Deposition through such a mask results in background-free nanolines, and thus gives a direct measurement of the total deposited layer thickness. A SEM-scan of the mask and an AFM-scan of the Fe-lines grown through the mask are shown in Fig. 6. The FWHM width of the structures is about $150 \mathrm{~nm}$. From the height of the deposited structures the deposition rate is measured. The measured deposition rate varies between 3 and $7 \mathrm{~nm} /$ hour, depending on the exact temperature and filling of the crucible. In the evaluation of the deposited structures, the calibration of the deposition rate has been performed under conditions as close to the specific deposition experiment as possible.

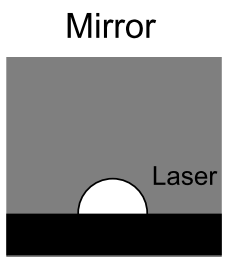

Substrate

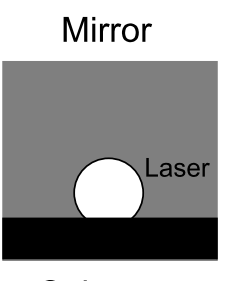

Substrate
Fig. 5 The cut-off of the laser beam by the substrate. Left: the laser beam is cut in half by the substrate (50\% cut-off). Right: only $10 \%$ of the laser beam is cut off by the substrate (10\% cut-off)

\section{Results}

We have deposited nanoline arrays of Fe with both negative and positive detuning of the light and with substrates positioned both at $50 \%$ cut-off and at $10 \%$ cut-off. The dependence on the light intensity of the height and width of the nanolines has been investigated, as well as the ratio of line height to the average layer thickness. Since we have an AOM with a fixed RF-frequency of $150(2 \pi) \mathrm{MHz}$, our measurements are limited to blue- and red-detuned focusing at this frequency shift from resonance.

On each deposited sample, the height and width of the nanolines has been measured as a function of position transverse to the laser beam axis (i.e., along the $y$-axis). Figure 7 depicts the geometry. As the laser beam has a Gaussian profile, each $y$-position is characterized by a different light intensity.

In Fig. 8 a $4 \times 1.5 \mu \mathrm{m}$ AFM scan of a typical sample after deposition is shown. In 2 hours deposition time we grow structures up to heights of $6 \mathrm{~nm}$. Figure 9 shows a cross section through the scan over a range of $1 \mu \mathrm{m}$. The distance between consecutive lines (the pitch of the modulation) is equal to $\lambda / 2=186 \mathrm{~nm}$.

Figure 10 shows a typical profile of the height of the nanolines as a function of transverse position. The intensity

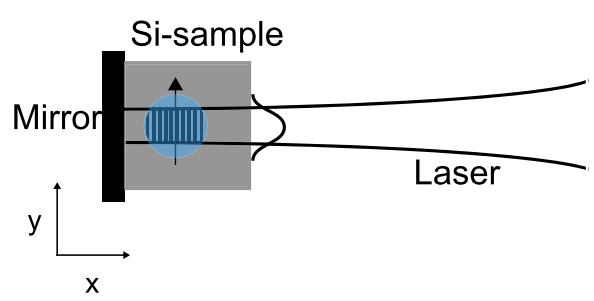

Fig. 7 Deposition assembly viewed from the direction of the incoming atomic beam. Nanolines are deposited on the substrate where the atomic beam intersects the standing light field (round spot). The height and width of the nanolines are investigated as a function of position along the nanolines. Since the laser beam is Gaussian, each position corresponds to a specific light intensity
Fig. 6 Left: SEM-scan of the $\mathrm{SiN}$ mask. The pitch of the lines is $744 \mathrm{~nm}$. Right: AFM-scan of the deposited Fe-lines through the mask. Deposition time is two hours

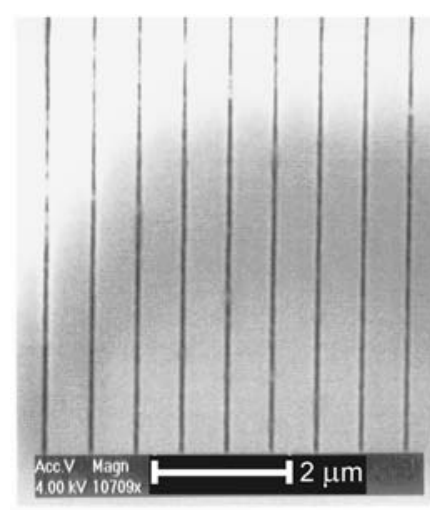

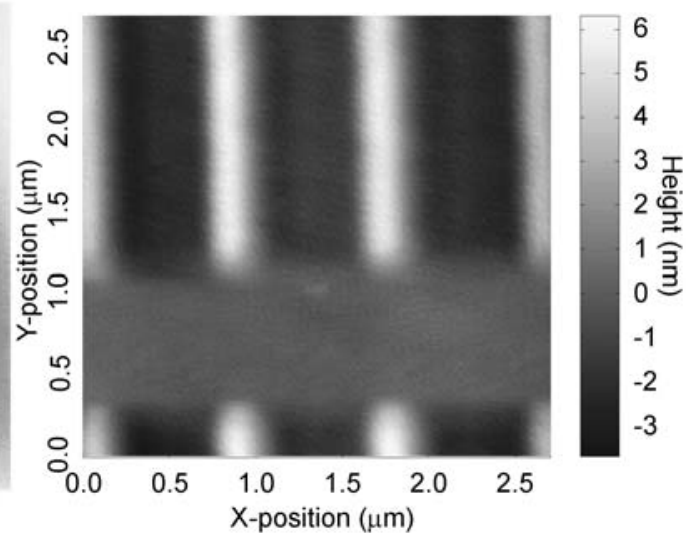




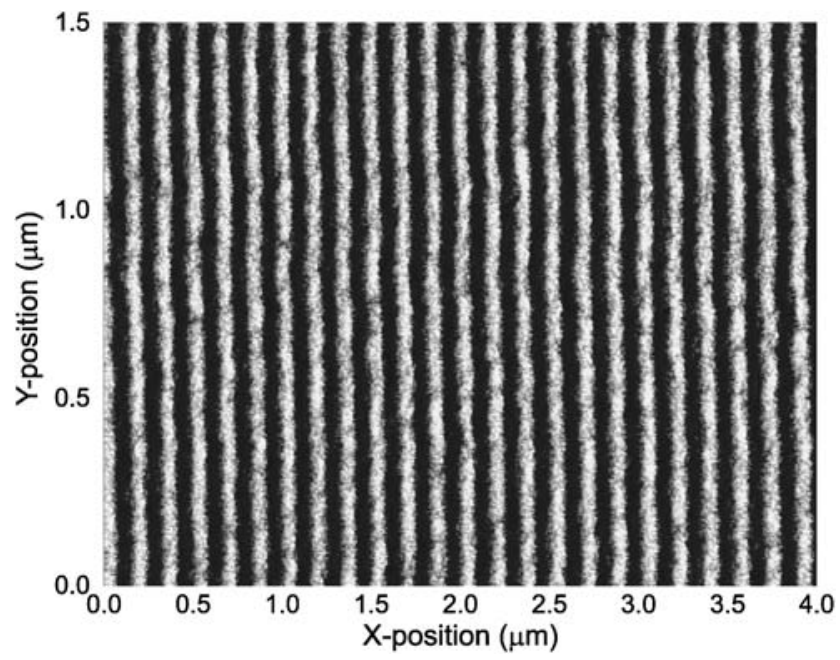

Fig. 8 Example of a $4 \times 1.5 \mu \mathrm{m}$ AFM-scan of the nanolines. The height (grey) scale ranges over $6 \mathrm{~nm}$

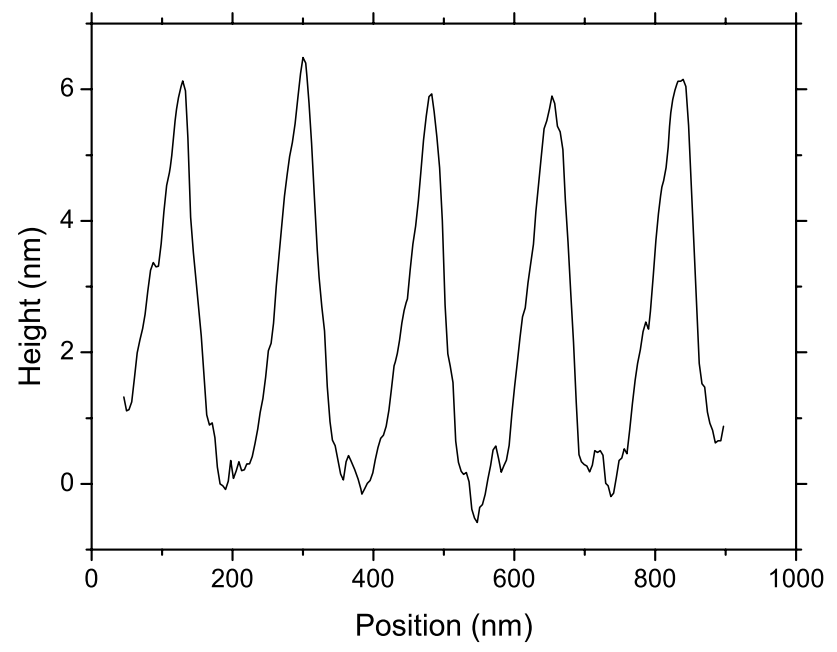

Fig. $91 \mu \mathrm{m}$ line-scan, averaged over $40 \mathrm{~nm}$ in the $Y$-direction, of the same sample shown in Fig. 8. The deposition time is 2 hours. The height of the structures in this image is $6 \mathrm{~nm}$ and their FWHM width approximately $60 \mathrm{~nm}$. The laser power is $P=50 \mathrm{~mW}, 10 \%$ of the laser beam is cut-off by the Si substrate

profile of the laser beam is shown as well. For high intensities, the height of the nanolines is largely independent on the light intensity both in experiment and simulation: the height profile does not follow the Gaussian shaped laser intensity profile, but it has a broad flat top. The height of the potential shown in Fig. 2 follows, in the high intensity limit, from the square-root of the intensity. Increasing the intensity will move the focus over the $z=0$ point towards negative $z$-values. Since the lens, especially in the blue-detuned case, has a large focal depth, the atoms remain focused at the $z=0$ position, even for higher intensities, resulting in the flat top of the height profile.

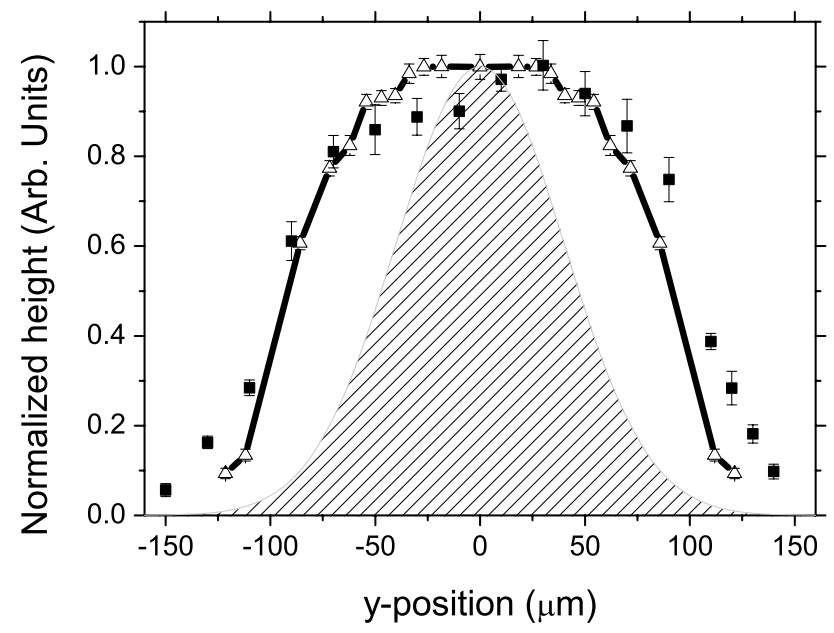

Fig. 10 Typical height profile as a function of position along the lines for a blue-detuned 50\% cut-off laser beam. Both simulation data and experimental data are normalized to their maximal height. The squares are the measurements, the triangles are the simulation. The patterned profile reflects the dependence of the standing-wave intensity on position. The laser power is $P=50 \mathrm{~mW}$

The results of the simulations are shown as well in Fig. 10. Both curves are normalized to the same height. The agreement between the experimental data and the results of the simulations is good when we look at the shape of the profile. At low intensities, the measurements deviate somewhat from the simulations. This may be caused by the nonGaussian tails in the spatial profile of the laser beam used in the experiment.

Experimental data and simulation results on the width of the structures as function of the position on the substrate are shown in Fig. 11. For high intensities, blue detuning results in slightly narrower lines compared to red detuning in the experiment. This difference is more pronounced in the simulations, and results from different focusing potentials (Fig. 2). The match between measured and simulated widths is better in the $10 \%$ cut-off configuration than in the 50\% cut-off configuration. In the latter configuration, the predicted widths are smallest, but the measured widths are similar to those in the $10 \%$ cut-off configuration. At the center of the laser beam profile and blue detuning, the measured FWHM width is $50 \mathrm{~nm}$ whereas the predicted width is around $35 \mathrm{~nm}$. In our earlier experiments, where the incoming atomic beam was collimated by laser cooling to a divergence $\alpha_{\mathrm{RMS}}=0.17 \mathrm{~m} / \mathrm{s}$ (compared to $\alpha_{\mathrm{RMS}}=0.38 \mathrm{~m} / \mathrm{s}$ in this experiment), the experimental width for the same laser intensity and detuning was almost equal $(55 \mathrm{~nm})$. Here, the simulations predict a width of $20 \mathrm{~nm}$. Evidently, the divergence of the atomic beam is, in both experiments, not the limiting factor for the width of the structures.

The relation between the divergence of the atomic beam and the simulated structure width is illustrated in Fig. 12. In this figure, the results for both the geometrically de- 
Fig. 11 FWHM of the lines as a function of position along the lines for blue and red detuning. The squares are the measurements. Simulations are indicated by the triangles. In the lower right graph, the laser intensity profile as used in the simulations is shown

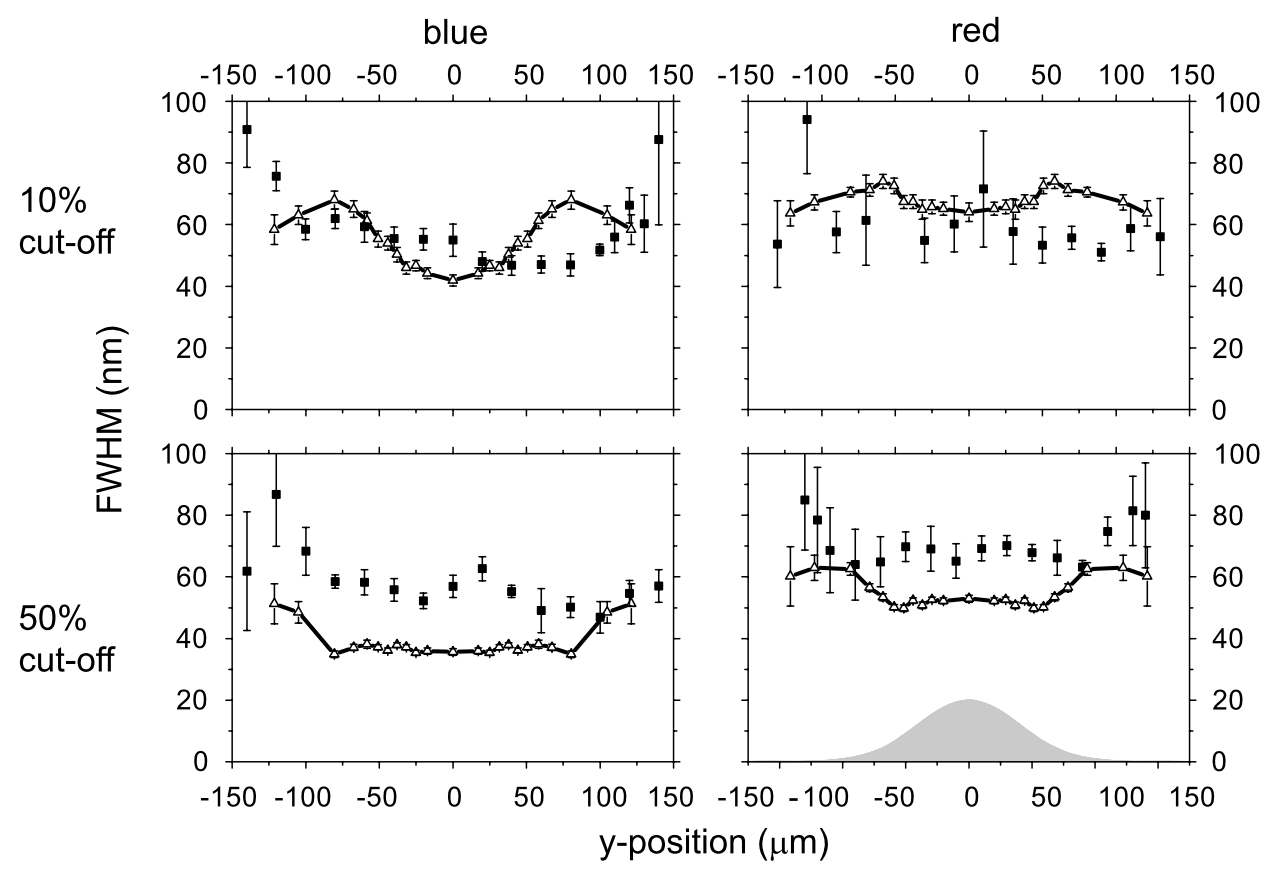

The presence of broadening mechanisms that are not accounted for in the simulations can explain the discrepancy. One such mechanism is the effect of diffraction of the laser beam by the substrate. This might be significant for the $50 \%$ cut-off configuration, where the substrate intercepts the laser beam at maximum intensity. The diffraction, which is extremely sensitive to the surface condition and to the precise orientation of the substrate, will distort the wavefronts of the standing light wave and generally lead to broader structures. Monitoring of the diffraction by imaging of the reflected laser beam profile has unfortunately not been implemented in the present experiment, but will be incorporated in future experiments.

A second broadening mechanism that is not included in the simulations is post-deposition broadening by surface diffusion of the deposited $\mathrm{Fe}$ atoms. This has been suggested in the literature [19-21] as a cause for earlier observed discrepancies between experimental and predicted structure widths $[22,23]$. Surface diffusion is very sensitive to the exact surface and vacuum conditions and therefore difficult to include in the simulations. However, it can definitely cause broadening on the order of the observed discrepancies [21].

Determining the ratio of the height of the nanolines to the average thickness of the Fe layer requires the calibration of the overall deposition rate as discussed in Sect. 3. Although this calibration is not very accurate, the result is that in our experiment the ratio for all experimental conditions is approximately 0.5 for the lines at maximum laser intensity (the center of the height profile as shown in Fig. 10).

To obtain the same ratio from the simulations we assume that $50 \%$ of the atoms are in the ${ }^{5} \mathrm{D}_{4}$ ground state, which is when it exceeds $0.6 \mathrm{mrad}$. 
Table 1 Simulation results of the ratio of the height of the lines to the average layer thickness

\begin{tabular}{lll}
\hline & Red & Blue \\
\hline $50 \%$ cut-off & 1.25 & 1.25 \\
$10 \%$ cut-off & 0.60 & 0.70 \\
\hline
\end{tabular}

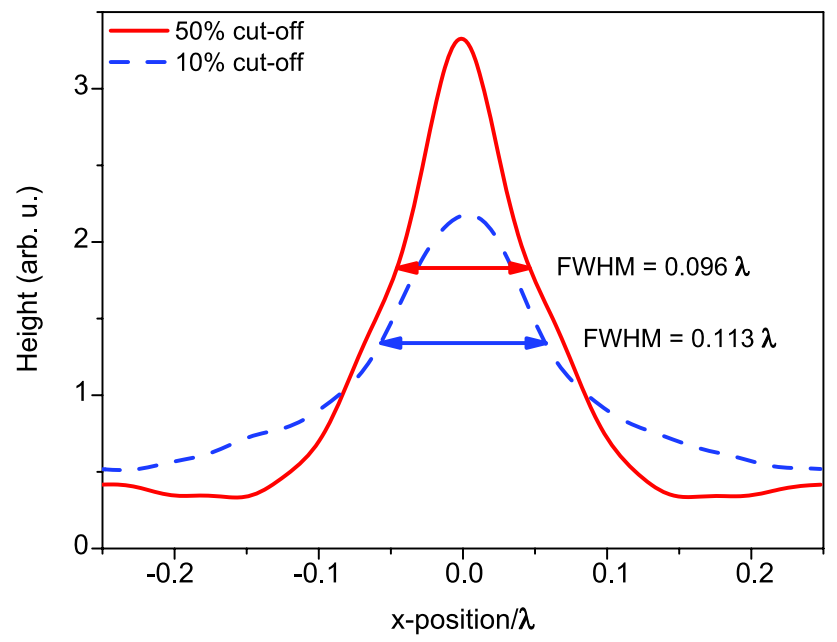

Fig. 13 Simulated profile of the deposited lines (blue detuning, at the center of the laser beam profile). Solid line: $50 \%$ cut-off configuration. Dashed line: $10 \%$ cut-off configuration. The FWHM width of the line profiles is indicated by the arrows

the thermal occupation of the ground state at a source temperature of $2,000 \mathrm{~K}$. The other $50 \%$ of the ${ }^{56} \mathrm{Fe}$ atoms, as well as the other isotopes, are not affected by the focusing light field and only contribute to the background layer. In Table 1 the ratio between simulated line heights and the average Fe layer thickness at maximum laser intensity is listed. For the $10 \%$ cut-off setting the simulated ratio is close to the experimental value of 0.5 . For the $50 \%$ cut-off case, the simulated ratio is much larger.

The large difference in the simulated ratios for the two cut-off settings cannot be explained solely by the difference in line widths, which is approximately $20 \%$. The most important factor here is the difference in the shape of the deposited structure profile. For the $10 \%$ cut-off setting, the profile has a narrow but low central peak with broad wings, whereas for $50 \%$ cut-off the central peak is higher and has less prominent wings. The latter situation leads to a larger ratio of peak height to average layer thickness. The difference in shape of the simulated profiles is illustrated in Fig. 13.

As indicated earlier, the discrepancy between measurement and simulation for the 50\% cut-off case is also present in the widths of the lines, which is larger in the experiment than in the simulations. Both discrepancies can be attributed to the same broadening mechanisms mentioned before.

\section{Conclusions}

By direct-write atom lithography Fe nanolines are deposited with a pitch of $186 \mathrm{~nm}$ and a FWHM width of $50 \mathrm{~nm}$, without the use of laser collimation techniques. Sufficient collimation is obtained by strong geometrical collimation, produced by the relatively large distance $(650 \mathrm{~mm})$ between Fesource and sample compared to the $1 \mathrm{~mm}$ source nozzle. In this way the divergence of the atoms arriving at the sample is reduced to $0.4 \mathrm{mrad}$ RMS.

Experiments and simulations are performed with the focusing standing light wave cut in half by the substrate and with a $10 \%$ cut-off by the substrate. Experiments and simulations are in good agreement with respect to the height of the nanolines. The experimental width of the nanolines corresponds well with the simulations for the $10 \%$ cut-off case. For the 50\% cut-off case the measured width of the lines is slightly larger than the simulated value. Also, the measured height of the lines versus average deposited layer thickness ratio is lower than in the simulations in that case. We attribute this discrepancy to diffraction of the light beam by the substrate and small misalignments of the standing light wave.

The fact that direct-write atom lithography can be applied without the use of laser cooling techniques opens the way to many new applications. Except for a small number of elements with a strong closed-level transition in an easily accessible wavelength region, laser cooling can add a serious and sometimes insurmountable complication to atom lithography. Past efforts to apply the technique to atoms that are interesting from a technological viewpoint (e.g., gallium or indium in view of III-V semiconductor applications) have concentrated on the laser collimation as a first step. These efforts have succeeded in the challenging goal of achieving laser collimation $[9,11]$, but have not yet resulted in the production of nanostructures. The presented results show that, by using an high-flux beam source and relying on geometrical collimation, laser collimation can be bypassed completely.

Acknowledgements This work is part of the research program of the Foundation for Fundamental Research on Matter (FOM), which is financially supported by the Netherlands Organisation for Scientific Research (NWO).

Open Access This article is distributed under the terms of the Creative Commons Attribution Noncommercial License which permits any noncommercial use, distribution, and reproduction in any medium, provided the original author(s) and source are credited.

\section{References}

1. G. Timp, R.E. Behringer, D.M. Tennant, J.E. Cunningham, Phys. Rev. Lett. 69, 1636 (1992) 
2. J.J. McClelland, R.E. Scholten, E.C. Palm, R.J. Celotta, Science 262, 877 (1993)

3. U. Drodofsky, J. Stuhler, Th. Schulze, M. Drewsen, B. Brezger, T. Pfau, J. Mlynek, Appl. Phys. B 65, 755 (1997)

4. R.W. McGowan, D.M. Giltner, S.A. Lee, Opt. Lett. 20, 2535 (1995)

5. R. Ohmukai, S. Urabe, M. Watanabe, Appl. Phys. B 77, 415 (2003)

6. E. te Sligte, B. Smeets, K.M.R. van der Stam, R.W. Herfst, P. van der Straten, H.C.W. Beijerinck, K.A.H. van Leeuwen, Appl. Phys. Lett. 85, 4493 (2004)

7. G. Myszkiewicz, J. Hohlfeld, A.J. Toonen, A.F. Van Etteger, O.I. Shklyarevskii, W.L. Meerts, Th. Rasing, E. Jurdik, Appl. Phys. Lett. 85, 3842 (2004)

8. B. Smeets, R.W. Herfst, L.P. Maguire, E. te Sligte, P. van der Straten, H.C.W. Beijerinck, K.A.H. van Leeuwen, Appl. Phys. B 80, 833 (2005)

9. S.J. Rehse, K.M. Bockel, S.A. Lee, Phys. Rev. A 69, 063404 (2004)

10. H. Leinen, D. Glässner, H. Metcalf, R. Wynands, D. Haubrich, D. Meschede, Appl. Phys. B 70, 567 (2000)

11. B. Klöter, C. Weber, D. Meschede, H. Metcalf, Phys. Rev. A 77, 033402 (2008)

12. T. Fujii, H. Kumgai, K. Midorikawa, M. Obara, Opt. Lett. 25, 1457 (2000)
13. D. Meschede, H. Metcalf, J. Phys. D: Appl. Phys. 36, R17 (2003)

14. M.K. Oberthaler, T. Pfau, J. Phys.: Condens. Matter 15, R233 (2003)

15. J. Dalibard, C. Cohen-Tannoudji, J. Opt. Soc. Am. B 2, 1707 (1985)

16. C.G.C.H.M. Fabrie, L.P. van Dijk, B. Smeets, T. Meijer, K.A.H. van Leeuwen. Submitted to J. Phys. D: Appl. Phys.

17. R.C.M. Bosch, H.C.W. Beijerinck, P. van der Straten, K.A.H. van Leeuwen, Eur. Phys. J. Appl. Phys. 18, 221 (2002). The atomic beam source described in this paper is used in thermal mode

18. B. Smeets, R.C.M. Bosch, P. van der Straten, E. te Sligte, R.E. Scholten, H.C.W. Beijerinck, K.A.H. van Leeuwen, Appl. Phys. B 76, 815 (2003)

19. E. Jurdík, Th. Rasing, H. van Kempen, C.C. Bradley, J.J. McClelland, Phys. Rev. B 60, 1543 (1999)

20. R.M. Bradley, A. Eschmann, S.A. Lee, J. Appl. Phys. 88, 3316 (2000)

21. E. te Sligte, K.M.R. van der Stam, B. Smeets, P. van der Straten, R.E. Scholten, H.C.W. Beijerinck, K.A.H. van Leeuwen, J. Appl. Phys. 95, 1749 (2004)

22. R.E. Behringer, V. Natarajan, G. Timp, Appl. Surf. Sci. 104/105, 291 (1996)

23. W.R. Anderson, C.C. Bradley, J.J. McClelland, R.J. Celotta, Phys. Rev. A 59, 2476 (1999) 